\title{
Food process automation
}

\author{
Kuanglin Chao $\cdot$ Moon S. Kim $\cdot$ Renfu Lu
}

Published online: 11 February 2009

(C) Springer Science+Business Media, LLC 2009

For the conversion of agricultural commodities into consumable and marketable food products, science and technology are now enabling amazing advances in food process automation. Mechanization has achieved startling reductions in the labor requirements of processing operations between farm and fork, and now automation has begun reducing the considerable human sensory input component involved in many food processing operations.

Automation allows processors to satisfy high-volume demand and distribution needs with greater efficiency while also providing greater flexibility and responsiveness in maintaining and improving food quality and safety.

Food safety remains bigger than ever in consumer awareness, with incidents of contamination and outbreaks of food borne illness rooted in problems ranging across the food system, from the fields and livestock of producers to the operations of processors, distributors, and retail establishments. Solutions to many of these problems can be effectively implemented through automation, provided that new sensing technologies are appropriately developed for specialized applications in the food production and distribution system, and that both technical knowledge and understanding of food safety and security needs are shared

K. Chao $(\bowtie) \cdot$ M. S. Kim

Environmental Microbial and Food Safety Lab, USDA, ARS, Beltsville, MD, USA

e-mail: kevin.chao@ars.usda.gov

M. S. Kim

e-mail: moon.kim@ars.usda.gov

R. Lu

Sugarbeet and Bean Research Unit, USDA, ARS, East Lansing, MI, USA

e-mail: renfu.lu@ars.usda.gov between those who develop the automation technologies and those who will implement them.

Food process automation will depend on both fundamental and applied research in sensor technologies for process control and monitoring, as well as data management strategies for product tracking and traceability, packaging and distribution, and sanitation and inspection.

This special issue of Sensing and Instrumentation for Food Quality and Safety highlights some of the new technologies, ideas and research findings currently being developed for food process automation by researchers from academia, government, and industry worldwide. The articles in this special issue cover recent advances in different aspects of food process automation, and also provide overviews of spectroscopy-based monitoring technologies and of systems, standards, and interfaces for automated food processing operations.

Ghoush and Jayas first present the basic working principles of a broad spectral range of techniques currently used for automated food process monitoring, ranging from ultraviolet spectroscopy to nuclear magnetic resonance. Significant quality and safety improvements in food processing have come about from the integration of these nondestructive techniques.

Next is a broad review by Mahalik of systems, standards, and interfaces for advanced automation in the food process industry, covering a variety of technologies for processing and packaging and the current state of the art.

The use of radio frequency identification (RFID) is a significant and growing trend in food distribution system management. Amador et al. present the use of RFID technologies for process traceability, with particular focus on real-time monitoring the environment of food products along the supply chain to enable rapid decision making and the capture of long duration temperature profiles. Laniel 
et al. present a study for the effectiveness of using RFID tags for real-time tracking on large enclosed containers that may be affected by variables of frequency and antenna position in addition to specific product composition.

Hyperspectral and multispectral imaging is another technology finding many uses in food quality and safety applications. With the combination of nondestructive imaging and chemical/biological sample characterization, suitable applications include inspection and monitoring of both food products and the systems that process them. Jun et al. present an investigation into the use of hyperspectral fluorescence imaging for assessing stainless steel surfaces for bacterial biofilms that can present food safety risks and indicate problems in process sanitation management. Cui et al. report that multispectral imaging with three wavebands could be used to quantitatively detect the infection and severity of soybean rust, one of the most destructive diseases in soybean production.

Examples of the application of such food process automation technologies are presented for sanitation, food quality, and food safety. Patel addresses the use of reactive oxygen in meat processing environments for air decontamination to minimize the potential contamination of product from airborne bacteria. Fagan et al. present the use of visible/near-infrared light backscattering for the prediction of curd moisture and whey fat content in cheese production. Yang et al. present an automated machine vision system based on line-scan imaging for wholesomeness inspection of young chickens on high-speed commercial processing lines that can increase processing efficiency and reduce labor while maintaining and improving product safety. Kondo presents advanced commercial robotic systems for automating the presentation of fresh fruit for inspection and the quality grading of the fruit, thus reducing labor requirements with increased objectivity and consistency, and enabling product quality traceability and greater quality-based product sales.

For this special issue of Sensing and Instrumentation for Food Quality and safety, we are grateful to all the research contributors and also to the referees for reviewing these papers. We hope that our readers will find this sampling of current and developing work in food process automation technologies useful and informative. 